\title{
CONTRIBUTION PATERNELLE DE GENOTYPES SAUVAGES D'HEVEA (Hevea brasiliensis Muell. Arg.) EN POLLINISATION LIBRE DANS UN VERGER A GRAINES
}

\author{
Y. J. LIDAH' , A.S.-P. N'GUETTA ${ }^{1}$, G. M. RODIER ${ }^{3}$, C.-D. ANDRE² et M. SEGUIN² \\ ${ }^{1}$ Laboratoire de génétique Université de Cocody 22 Bp 582 Abidjan 22. E-mail : justinlidah@yahoo.fr \\ ${ }^{2}$ CIRAD Cultures Pérennes, Programme Hévéa Avenue Agropolis 34398 Montpellier Cedex 5 France \\ ${ }^{3}$ CIRAD AGETROP, Programme Cacao Avenue Agropolis 34398 Montpellier Cedex 5 France
}

\begin{abstract}
RESUME
La Côte-d'Ivoire dispose d'une collection d'hévéas sauvages structurée en deux groupes génétiques: AM1 et AM2. L'intégration de cette collection dans la stratégie de création variétale est envisagée par pollinisation libre dans des vergers à graines. Pour comprendre le brassage génétique au sein de cette collection, la contribution paternelle de 50 génotypes greffés en verger à graines a été étudiée à l'aide de 8 loci microsatellites à travers l'analyse de la paternité sur 2 années successives. L'étude a montré un brassage génétique déséquilibré entre les génotypes issus de la collection d'hévéas sauvages. La majorité des génotypes a très faiblement participé à la pollinisation. Sur les deux années, leur contribution paternelle a varié de 0,21 à $22,7 \%$. Cependant, 4 génotypes ont dominé particulièrement la pollinisation avec une contribution paternelle cumulée d'au moins $40 \%$. La contribution paternelle a été variable entre les génotypes, et également entre les groupes AM1 et AM2. Le régime de reproduction de la collection sauvage est donc non panmictique. Aucune variation inter-annuelle de la contribution paternelle des génotypes à la pollinisation a été observée. Ce qui dénote d'une caractéristique clonale de ce facteur.
\end{abstract}

Mots clés : Hevea brasiliensis, système de reproduction, verger à graines, pollinisation libre, contribution paternelle, Côte-d'Ivoire

\author{
ABSTRACT \\ PATERNAL CONTRIBUTION OF WILD RUBBER TREES (Hevea brasiliensis MUell. arg.) IN OPEN POLLINATION \\ INA SEEDS ORCHARD
}

The ivoirian rubber breeding program has a collection of wild genotypes structured in two genetic groups: AM1 and AM2. The use of this wild collection for cloning purposes is considered through open pollination in seeds orchard. To assess the genetic brewing within this collection, the paternal contribution of 50 genotypes transplanted in a seeds orchard, has been studied with 8 microsatellites loci through paternity analysis in 2 years successively. Thie study shows that genetic brewing was unbalance within the collection of wild rubber trees. Nearly all the genotypes, participated very weakly in the pollination process. During the two years, their paternal contribution varied from 0.21 to $22.7 \%$. However, 4 genotypes, particularly dominated the pollination process. Their cumulated paternal contribution was estimated at $40 \%$ for the least. The paternal contribution was very variable between both genotypes and $A M 1$ and $A M 2$ genetic groups. Thus, the reproduction system of the wild rubber tree collection is no panmictic. No Inter annual variation of the genotypes paternal contribution occured. Thus, paternal contribution appear to be a clonal characteristic of rubber tree species.

Keywods : Hevea brasilliensis, mating system, seeds orchard, open pollination, paternal contribution, Côted'Ivoire 


\section{INTRODUCTION}

La contribution paternelle ou succès reproducteur mâle, est la mesure de la participation d'un géniteur mâle à des croisements (Yacine et Lumaret, 1998). C'est un paramètre important en pollinisation libre puisqu'il permet d'apprécier la qualité du brassage génétique entre différents génotypes en présence. Lorsqu'une population d'arbres est laissée en pollinisation libre dans un verger à graines, l'égalité des contributions paternelles suggère un mode de reproduction panmictique, nécessaire à l'obtention de gains génétiques substantiels (Baradat et al., 1984). Le mode de reproduction qui représente la loi de transmission des gènes d'une génération à une autre (Baciellerie, 1994) peut donc être défini par rapport aux contributions paternelles.

En Côte-d'Ivoire, l'amélioration de la population sauvage d'hévéa (Hevea brasiliensis), essence forestière originaire d'Amazonie brésilienne et productrice de latex, a été envisagée par pollinisation libre en verger à graines. En effet, avec l'introduction de génotypes sauvages prospectés au Brésil en 1981, la Côte-d'Ivoire dispose désormais de deux populations d'hévéa à performances complémentaires :

- la population sauvage ou population amazonienne «Am» présente une faible productivité, une résistance aux maladies des feuilles et une bonne vigueur (Clément-Démange et al., 1995 ; 1997) ;

- Ia population améliorée, ou population Wickham «W» possède une plus grande valeur productive avec une vigueur moyenne, mais qui est sensible aux maladies des feuilles (Legnaté et Clément-Demange, 1991a).

L'intégration des génotypes sauvages dans la stratégie d'amélioration génétique de l'hévéa est nécessaire, puisque les progrès génétiques réalisés avec la population cultivée s'amenuisent, à cause de l'étroitesse de la variabilité génétique et de la trop forte consanguinité des géniteurs recombinants (Ho, 1979). Après la structuration de la population sauvage en deux groupes génétiques: AM1 et AM2 (Chevallier, 1988), des croisements inter populations ont été réalisés. Ces premiers croisements ont montré l'expression d'une vigueur hybride au niveau de la croissance et le transfert de la résistance aux maladies des feuilles des parents sauvages aux hybrides. Il a été noté également une amélioration de la productivité des hybrides par rapport aux parents sauvages de faibles productivité (Légnaté et Clément-Demange, 1991b).

Ainsi, compte tenu de l'additivité des effets géniques sur les caractères chez l'hévéa, l'amélioration du niveau de production de la population sauvage par sélection récurrente réciproque en verger à graines a été suggérée avant de les croiser avec des clones cultivés (Baudoin et al., 1997). Pour ce faire, des études, au sein de la population sauvage, sont réalisées pour définir les conditions d'une sélection récurrente réciproque performante.

La présente étude, réalisée sur 2 années successives, vise à étudier la qualité du brassage génétique de la population sauvage par la détermination de la contribution paternelle de génotypes en pollinisation libre dans un verger à graines.

\section{MATERIEL ET METHODES}

\section{MATERIEL VEGETAL}

L'étude a été conduite en 1998 et en 1999 avec un matériel constitué d'une population parentale et ses descendants. La population parentale comprend 50 génotypes sauvages et un génotype cultivé mâle stérile, appelé GT1 (Leconte, 1984). Les génotypes sauvages ont été repartis équitablement entre les deux groupes génétiques: AM1 et AM2 (Tableau 1), mis en évidence par la structuration de leur variabilité génétique (Chevallier, 1988) pour réaliser, à priori, un brassage inter groupe de cette population. Toute la population parentale a été greffée dans un verger à graines, à raison de 2 à 8 individus pour les génotypes sauvages et de 11 individus pour le génotype cultivé. Le verger à graines a été mis en place en 1992, selon un dispositif en quinconce sur une superficie de $8892 \mathrm{~m}^{2}$ (118 m x $\left.78 \mathrm{~m}\right)$. La distance entre deux rangées de plants est de $5,2 \mathrm{~m}$ tandis que deux arbres voisins sur une même rangée sont séparés par $6 \mathrm{~m}$. Le verger à graines a été établi dans la station expérimentale café-cacao du Centre National de Recherche Agronomique (CNRA), loin de toute plantation d'hévéa, afin d'empêcher de pollinisations extérieures. En 1998 l'essai comportait 287 arbres et 286 en 1999 à cause de la verse due au vent. Les arbres ont été 
identifiés par leur position géographique (ligne et rang) dans le verger à graines.

Les descendants ont été représentés par deux échantillons de plantules issues de la germination des graines obtenues par pollinisation libre de la population parentale sur les deux années. Les graines ont été récoltées directement sur les arbres pour garantir leur origine maternelle. En 1998, un échantillon de 467 plantules a été utilisé contre 404 en 1999. Ces descendants ont été organisés par famille d'arbre-mère ou de génotype qui est le regroupement de descendants d'au moins deux arbres-mères de même génotype (Tableau 2).

Tableau 1 : Génotypes d'hévéa du verger à graines planté à Divo en 1998.

Rubber tree genotypes from Divo seeds orchard planted in 1998.

\begin{tabular}{llcllc}
\hline \multicolumn{2}{l}{ Groupe génétique AM1 } & \multicolumn{3}{c}{ Groupe génétique AM2 } \\
\hline Codes & Génotypes & $\begin{array}{c}\text { Nombre } \\
\text { d'arbres }\end{array}$ & Codes & Génotypes & $\begin{array}{c}\text { Nombre } \\
\text { d'arbres }\end{array}$ \\
\hline 101 & AC/S/11/54 & 4 & 201 & MT/C/4/18 & 3 \\
103 & AC/S/8/118 & 6 & 203 & MT/IT/13/22 & 4 \\
106 & AC/B/19/97 & 6 & 204 & MT/IT/16/55 & 6 \\
107 & RO/OP/4/19 & 6 & 205 & MT/IT/18/4 & 6 \\
108 & AC/S/8/33 & 5 & 206 & MT/C/2/25 & 6 \\
110 & AC/S/8/59 & 6 & 207 & MT/C/4/37 & 6 \\
111 & AC/B/19/72 & 6 & 208 & MT/IT/16/58 & 6 \\
112 & RO/CM/10/130 & 4 & 209 & RO/PB/2/74 & 5 \\
114 & RO/CM/11/41 & 2 & 210 & MT/C/1/13 & 6 \\
115 & RO/CM/11/91 & 8 & 211 & MT/C/2/39 & 5 \\
117 & AC/S/12/57 & 5 & 212 & MT/C/2/50 & 5 \\
118 & AC/X/20/35 & 6 & 213 & MT/IT/17/26 & 5 \\
119 & AC/S/10/50 & 5 & 214 & MT/C/4/5 & 6 \\
120 & AC/S/10/53 & 5 & 215 & MT/C/5/11 & 6 \\
122 & RO/CM/11/29 & 5 & 216 & MT/IT/14/32 & 6 \\
123 & RO/CM/11/54 & 4 & 217 & RO/PB/2/29 & 5 \\
126 & AC/B/18/1 & 3 & 218 & MT/C/1/1 & 6 \\
130 & AC/S/9/16 & 6 & 219 & MT/C/4/22 & 6 \\
132 & RO/CM/10/109 & 4 & 220 & MT/C/5/26 & 6 \\
133 & RO/CM/12/10 & 5 & 221 & MT/C/6/15 & 5 \\
135 & RO/CM/12/21 & 4 & 222 & MT/C/9/2 & 5 \\
137 & AC/B/16/3 & 5 & 224 & MT/IT/17/10 & 4 \\
139 & AC/S/9/12 & 6 & MT/IT/17/15 & 6 \\
140 & AC/FA/6/29 & 6 & RO/PB/2/38 & 6 \\
142 & RO/CM/11/72 & 5 & MT/C/2/28 & 6 \\
\hline
\end{tabular}

Le génotype cultivé GT1 a été codé 300 il est représenté par 11 arbres et les arbres non conformes à leur présumé génotype sont au nombre de 13.

The code of the cultived genotype GT1 is 300; it was represented by 11 trees. There were 13 non compliant trees in the seeds orchard 
Tableau 2 : Descendances de Hevea brasiliensis analysées dans le verger à graines de Divo sur 2 années.

Hevea brasiliensis progenies analyzed in the seeds orchard of Divo for 2 years.

\begin{tabular}{|c|c|c|c|c|c|c|c|}
\hline \multicolumn{4}{|c|}{ Descendances analysées en 1998} & \multicolumn{4}{|c|}{ Descendances analysées en 1999} \\
\hline Génotypes & Groupe & $\begin{array}{c}\text { Nombre } \\
\text { arbres-mères }\end{array}$ & $\begin{array}{l}\text { Nombre de } \\
\text { descendants }\end{array}$ & Génotypes & Groupe & $\begin{array}{c}\text { Nombre } \\
\text { arbres-mères }\end{array}$ & $\begin{array}{l}\text { Nombre de } \\
\text { descendants }\end{array}$ \\
\hline 101 & & 1 & 12 & - & - & - & - \\
\hline 115 & & 3 & 38 & - & - & - & - \\
\hline 118 & & 2 & 37 & - & - & - & - \\
\hline 130 & AM1 & 1 & 20 & 140 & AM1 & 2 & 60 \\
\hline 140 & & 5 & 58 & Total AM1 & - & 2 & 60 \\
\hline Total AM1 & & 12 & 165 & - & - & - & - \\
\hline 204 & & 1 & 12 & 204 & AM2 & 0 & 0 \\
\hline 206 & & 1 & 16 & 206 & & 5 & 157 \\
\hline 210 & & 1 & 11 & 209 & & 0 & 0 \\
\hline 216 & & 0 & 0 & 210 & & 1 & 66 \\
\hline 218 & & 4 & 60 & 215 & & 0 & 0 \\
\hline 219 & & 2 & 21 & 216 & & 0 & 0 \\
\hline 221 & AM2 & 2 & 58 & 218 & & 0 & 0 \\
\hline 222 & & 1 & 28 & 220 & & 0 & 0 \\
\hline 224 & & 1 & 17 & 221 & & 1 & 63 \\
\hline Total AM2 & & 13 & 223 & 225 & & 0 & 0 \\
\hline 300 (GT1) & & 3 & 79 & Total AM2 & & 7 & 286 \\
\hline- & - & - & - & 300 (GT1) & & 2 & 58 \\
\hline Total 1998 & & 28 & 467 & Total & & 11 & 404 \\
\hline
\end{tabular}

\section{METHODE}

Tous les descendants échantillonnés et un arbre par génotype parental ont été analysés avec 8 loci microsatellites (Tableau 3). L'extraction de I'ADN (Acide Desoxyribonucléique) a été faite à partir de folioles matures et fraîches conservées à $-80^{\circ} \mathrm{C}$ selon le protocole au MATAB adapté de celui de Kit Quiagen. Ensuite, la PCR (Polymérase Chain Reaction) a permis d'amplifier les loci microsatellites dont les amorces ont préalablement été marquées au phosphore radioactif $\left({ }^{32} \mathrm{P}\right)$. La séparation des fragments d'ADN a été faite entre la cathode et l'anode sur un gel d'acrylamide concentré à $5 \%$, à une puissance constante de 60 watts. Après migration, des bandes ont été révélées par la méthode photochimique de coloration au nitrate d'argent.

Tableau 3 : Caractéristiques des loci microsatellites étudiés chez Hevea brasiliensis.

Characteristics of microsatellites loci used in Hevea brasiliensis.

\begin{tabular}{lcrlcc}
\hline Microsatellites & $\begin{array}{c}\text { Séquences } \\
\text { nucléotidiques }\end{array}$ & Amorces & $\begin{array}{c}\text { Taille } \\
\text { (paire de bases) }\end{array}$ & $\begin{array}{c}\text { Température } \\
\text { d'hybridation (min/max) }\end{array}$ \\
\hline T65 & $(\mathrm{GT})_{18}$ & 175 & 176 & 196 & $50 / 70$ \\
124 & $(\mathrm{GA})_{8} \mathrm{AA}(\mathrm{GA})_{6}$ & $25-2$ & $26-2$ & 166 & $50 / 70$ \\
A31 & $(\mathrm{GA})_{22}$ & 193 & 194 & 154 & $50 / 70$ \\
MnSOD & $(\mathrm{GA})_{16}$ & 5 & 6 & 200 & $55 / 72$ \\
A66 & $(\mathrm{GA})_{18}$ & 195 & 196 & 275 & $55 / 72$ \\
425 & $(\mathrm{CA})_{6}$ & 55 & 56 & 187 & $50 / 70$ \\
574 & $(\mathrm{TA})_{10}(\mathrm{GA})_{24}$ & $69-2$ & $70-2$ & 238 & $50 / 70$ \\
T67 & $(\mathrm{GT})_{21}$ & 185 & 186 & 120 & $50 / 70$ \\
\hline
\end{tabular}

$\mathbf{N b}$ : les chiffres en indice des séquences nucléotidiques sont les nombres de la répétition de la séquence

The number in index of the nucleotide sequences are the repetition number of the sequence 


\section{Analyse statistique des données}

L'analyse de la paternité selon Marchall et al. (1998) a permis de réaliser l'exploitation statistique des données microsatellites obtenues. La méthode utilisée a permis de rechercher le parent mâle de chaque plantule, étant donnée que le parent femelle est connu au départ. Cette méthode est basée sur l'approche de maximum de vraisemblance (Thompson, 1975 ; 1976 et Meagher 1986). A partir de l'analyse de la paternité, la contribution paternelle d'un génotype pollinisateur a été déterminée par le pourcentage de plantules dont ce génotype est le géniteur mâle. Afin de classer les génotypes pollinisateurs en fonction de leur contribution mâle, 4 classes ont été définies de façon arbitraire :

- la classe de génotypes faibles pollinisateurs, dont la contribution paternelle est comprise entre 1 et $2 \%$;

- la classe de génotypes pollinisateurs moyens, dont la contribution paternelle est comprise entre 2 et $5 \%$;

- la classe de génotypes grands pollinisateurs, dont la contribution paternelle est comprise entre 5 et $10 \%$;

- la classe de génotypes très grands pollinisateurs, dont la contribution paternelle est au moins égale à $10 \%$.

L'équilibre des contributions paternelles à la formation de la descendance d'un arbre-mère ou d'un génotype, a été testé selon la méthode de Streiff (1998) : Soit un parent mâle $p_{j}$ participant à la production de la descendance de cet arbre-mère et $f_{\text {l }}$ la probabilité d'avoir un descendant dont $\mathrm{p}_{j}$ est le parent mâle. A partir des $f_{j}$ des parents mâles, le nombre de parents mâles $P_{e f}$ participant théoriquement, pour des contributions paternelles équitables, à la création de la descendance, a été déterminé par la formule suivante : $P_{\text {ef }}=1 / \sum \mathrm{f}^{2}{ }_{j}$

L'expression $\left(\Sigma \mathrm{f}_{j}^{2}\right)$ représente la probabilité d'avoir deux descendants du même géniteur mâle ou la probabilité d'avoir deux pleins-frères dans la descendance de l'arbre-mère. Lorsque tous les parents mâles ont contribué également à la formation de la descendance d'un arbre donné, le nombre théorique de parents mâles $\mathrm{P}_{\text {ef }}$ est égal au nombre de parents mâles observés $\mathrm{P}_{o b}$. La comparaison entre le nombre de géniteurs mâles théoriques et celui des géniteurs mâles observés a été réalisée par le test du Khi2.

La régression linéaire a été utilisée pour apprécier l'évolution de la contribution paternelle d'un même génotype sur les deux années d'étude.

\section{RESULTATS}

\section{GENITEURS MALES DES PLANTULES ANALYSEES}

Les géniteurs mâles ayant participé à la formation des 467 plantules analysées en 1998 sont au nombre de 32 génotypes dont 12 du groupe AM1 et 20 du groupe AM2. En 1999, 40 géniteurs mâles ont été attribués à l'ensemble des 404 plantules analysées. Ces 40 géniteurs mâles sont constitués de 16 génotypes du groupe AM1 et de 24 génotypes du groupe AM2 (Tableau 4). Plus de géniteurs mâles du groupe AM2 participent donc à la pollinisation des arbres du verger à graines chaque année. Sur les deux années, un total de 42 géniteurs mâles sur les 51 pollinisateurs potentiels que compte le verger à graines ont été impliqués dans la formation des 871 plantules considérées. Ces 42 géniteurs mâles comptent 18 génotypes du groupe AM1 et 24 génotypes du groupe AM2. A l'exception des génotypes 110 et 118 du groupe AM1, tous les géniteurs mâles des deux groupes détectés en 1998 ont participé à la pollinisation en 1999. II est à noter également que le génotype GT1 n'a été le géniteur mâle d'aucune plantule.

\section{CONTRIBUTION PATERNELLE DES GENITEURS MALES}

La contribution paternelle individuelle des géniteurs mâles à la formation des plantules analysées a varie de 0,21 à 12,82 \% en 1998 et de 0,25 à $22,77 \%$ en 1999. Le regroupement des géniteurs mâles en fonction de leur contribution paternelle, a montré que la majorité des génotypes a eu une contribution paternelle individuelle très faible. Cette contribution paternelle a été inférieure à $2 \%$ pour 56,25 et $65 \%$ des géniteurs mâles qui ont été qualifiés de faibles pollinisateurs, respectivement en 1998 
et en 1999. Les classes de pollinisateurs moyens et de grands pollinisateurs comptent respectivement $21,88 \%$ et $15,62 \%$ de géniteurs mâles en 1998 et $22,5 \%$ et $10 \%$ en 1999. La classe de très grands pollinisateurs ayant au moins $10 \%$ de contribution paternelle renferme seulement 6,25\% des géniteurs mâles en 1998 et $4,16 \%$ des géniteurs mâles en 1999. La quasi-totalité des géniteurs mâles qualifiés de grands et de très grands pollinisateurs appartiennent au groupe AM2.

La contribution paternelle cumulée des géniteurs mâles $A M 1$ a été très inférieure à celle des génotypes AM2 (Figure 1). Les géniteurs mâles de AM1 ont contribué à la production de $16,34 \%$ des plantules contre $83,66 \%$ pour ceux de AM2 en 1998. Pendant la deuxième année d'étude, ces proportions ont été respectivement de $12,6 \%$ et de $87,39 \%$. La forte contribution paternelle du groupe AM2 a été due à quelques génotypes. II s'agit notamment de 4 clones codés 208, 206, 224 et 204 et à un degré moindre des génotypes 218 et de 221 (Figure 2). La contribution paternelle cumulée des 4 premiers clones cités a été estimée à $37,61 \%$ en 1998 et $42,33 \%$ en 1999. Deux de ces clones, le 204 et le 206, ont dominé particulièrement la pollinisation dans le verger à graines.

La relation entre la contribution paternelle des 42 géniteurs mâles et le nombre d'hévéas pollinisés par chacun d'entre eux a donné un coefficient de corrélation significatif de 0,78 (Figure 3). Ce résultat montre que la contribution paternelle d'un géniteur mâle serait en partie liée au nombre d'arbres qu'il pollinise. C'est-à-dire, le pollen des génotypes ayant une forte contribution paternelle est accepté par plusieurs arbres.

Le comportement sur les deux années des 42 géniteurs mâles révélés par l'analyse de la paternité a été étudié à l'aide d'une régression linaire avec un coefficient de 0,70 (Figure 4). Ce qui montre que la contribution paternelle d'un même géniteur mâle est stable quelle que soit l'année.

\section{VARIATION DE LA CONTRIBUTION PATERNELLE ENTRE LES POLLINISATEURS D'UN ARBRE OU D'UN GÉNOTYPE}

Aucune différence significative a été notée en 1998, entre les pollinisateurs observés et les pollinisateurs théoriques d'un arbre (Tableau 5). Les contributions paternelles ont donc été identiques entre les différents géniteurs mâles dans les familles d'arbre-mère pouvant être considérées comme des familles équilibrées. Au niveau des familles de génotype qui sont le regroupement des familles d'au moins deux arbres-mères de même génotype, la différence entre le nombre de géniteurs mâles observés et le nombre de géniteurs mâles théoriques a été significative (Tableau 6). Les géniteurs mâles qui ont participé à la production de la descendance d'un génotype ont eu des contributions paternelles déséquilibrées. Dans ce cas, la proportion de pleins-frères est importante pour les géniteurs mâles dont la contribution paternelle est la plus élevée. Dans ces familles, la possibilité de rencontrer une plantule formée par le pollen d'un géniteur mâle à grande contribution paternelle par rapport à un pollinisateur à faible contribution paternelle.

En 1999, les contributions paternelles n'ont pas été identiques entre les géniteurs mâles tant dans les familles d'arbre-mère que dans les familles de génotype (Tableau 7). Par rapport au descendants, le nombre de géniteurs mâles a été insuffisant dans les familles de génotypes. Le cas le plus expressif s'est rencontré en 1999 où 157 descendants de la famille du génotype 206, ont été produits grâce au pollen de 21 géniteurs mâles sur les 51 pollinisateurs potentiels du verger à graines.

Ces résultats montrent que les géniteurs mâles ont différemment contribué à la production des descendances dans le verger à graines. Ce qui induit la présence de fortes proportions de pleinsfrères dans les familles produites en pollinisation libre. Ce déséquilibre de contribution entre les pollinisateurs a semblé plus important en 1999 qu'en 1998 et serait la cause de croisements non panmictiques. 
Tableau 4 : Géniteurs mâles de plantules décelés par l'analyse de paternité avec 8 loci microsatellites chez Hevea brasiliensis

Males parents of the progenies revealed by paternity analysis of Hevea brasiliensis

\begin{tabular}{lll}
\hline & \multicolumn{1}{c}{ Géniteurs mâles AM1 } & \multicolumn{1}{c}{ Géniteurs mâles AM2 } \\
\hline $\begin{array}{l}\text { Echantillon de plantules } \\
1998\end{array}$ & $101,103,106,107,110,111,112,118,123$, & $201,203,204,205,206,207,208,209,210,211$, \\
\multirow{2}{*}{$\begin{array}{l}\text { Echantillon de plantules } \\
1999\end{array}$} & $126,135,142$ & $212,215,217,218,219,220,221,224,225,226$ \\
& $101,103,106,107,111,112,114,120,123$, & $201,203,204,205,206,207,208,209,210,211$, \\
& $126,130,132,133,135,140,142$ & $213,214,215,216,217,218,219,220,221$, \\
\hline
\end{tabular}

Les nombres désignent les codes des génotypes

Number are the codes of the genotypes

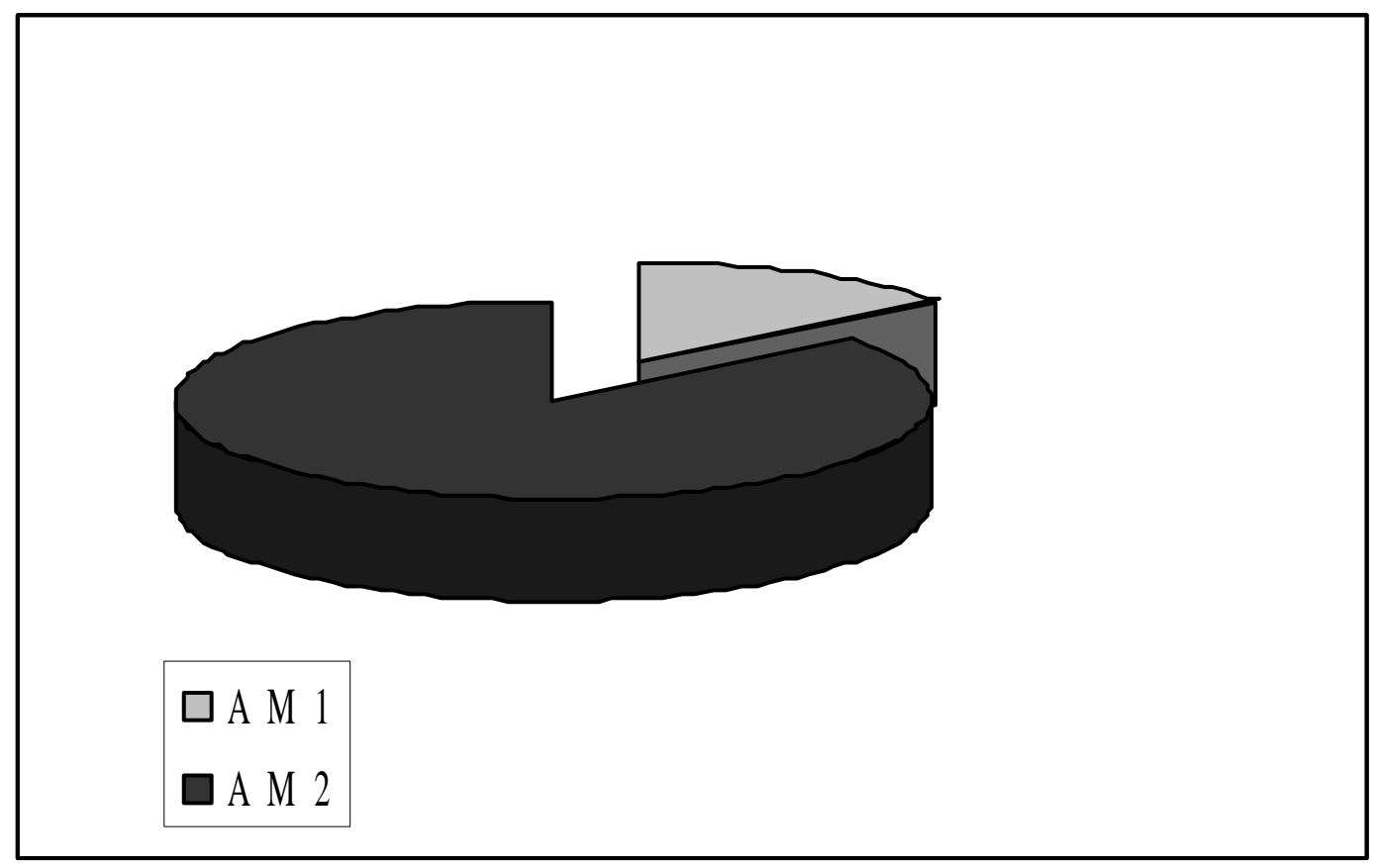

Figure 1 : Contribution paternelle cumulée de génotypes pollinisateurs des groupes AM1 et AM2 de Hevea brasiliensis sur 2 années.

Cumulated paternal contribution of the pollinators genotypes of the 2 genetics groups AM1 and AM2 of Hevea brasiliensis over a 2-year period. 


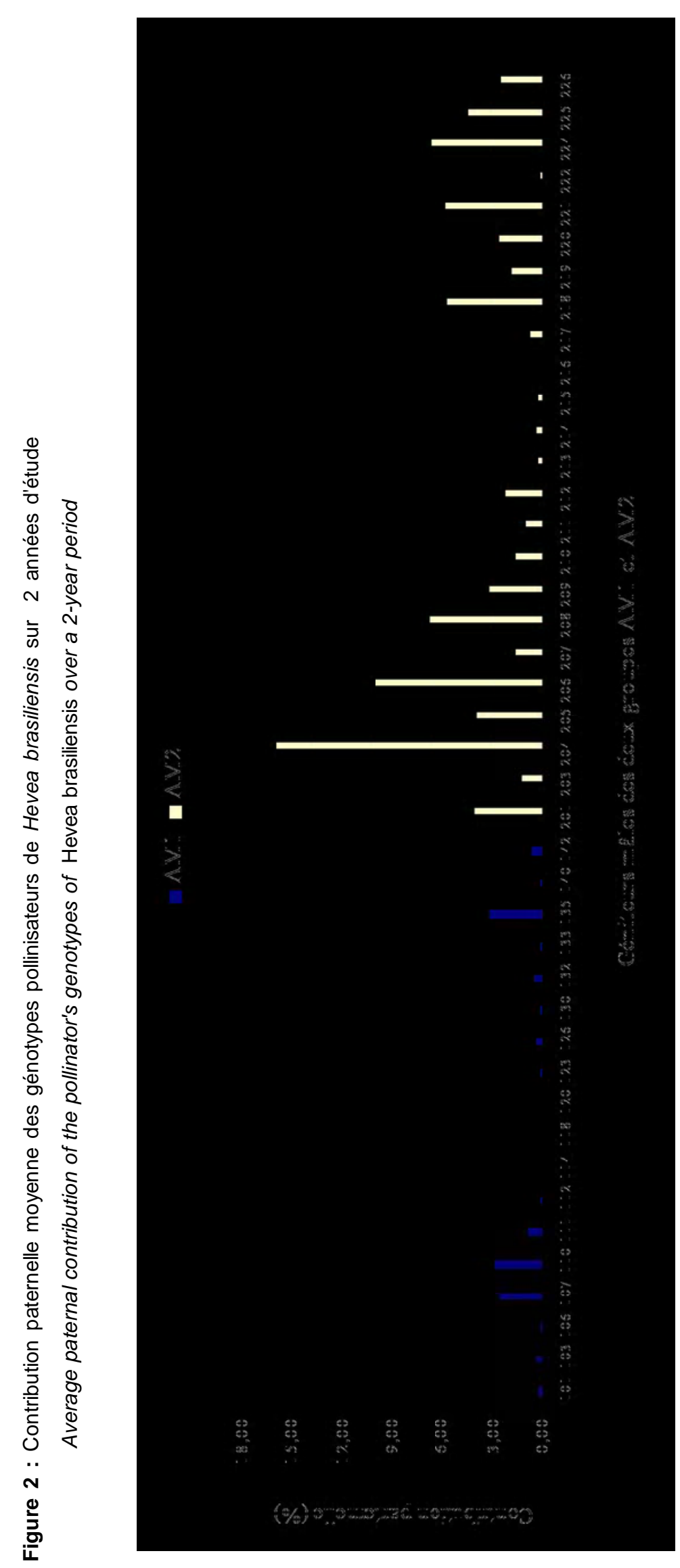




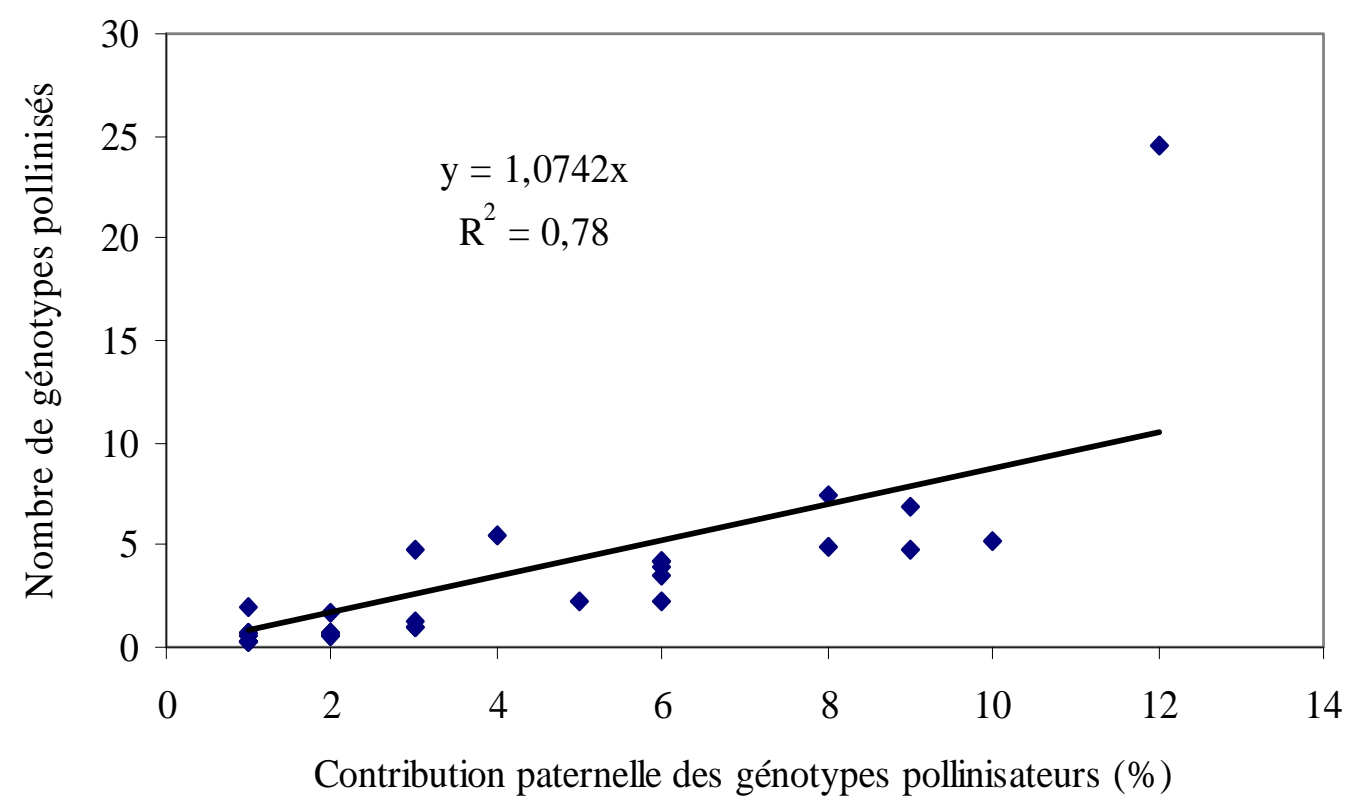

Figure 3 : Relation entre la contribution paternelle des génotypes d'hévéas et le nombre de géniteurs femelles pollinisés

Linear correlation between the paternal contribution of the rubber tree genotypes and the number of pollinated females over a 2-year period.

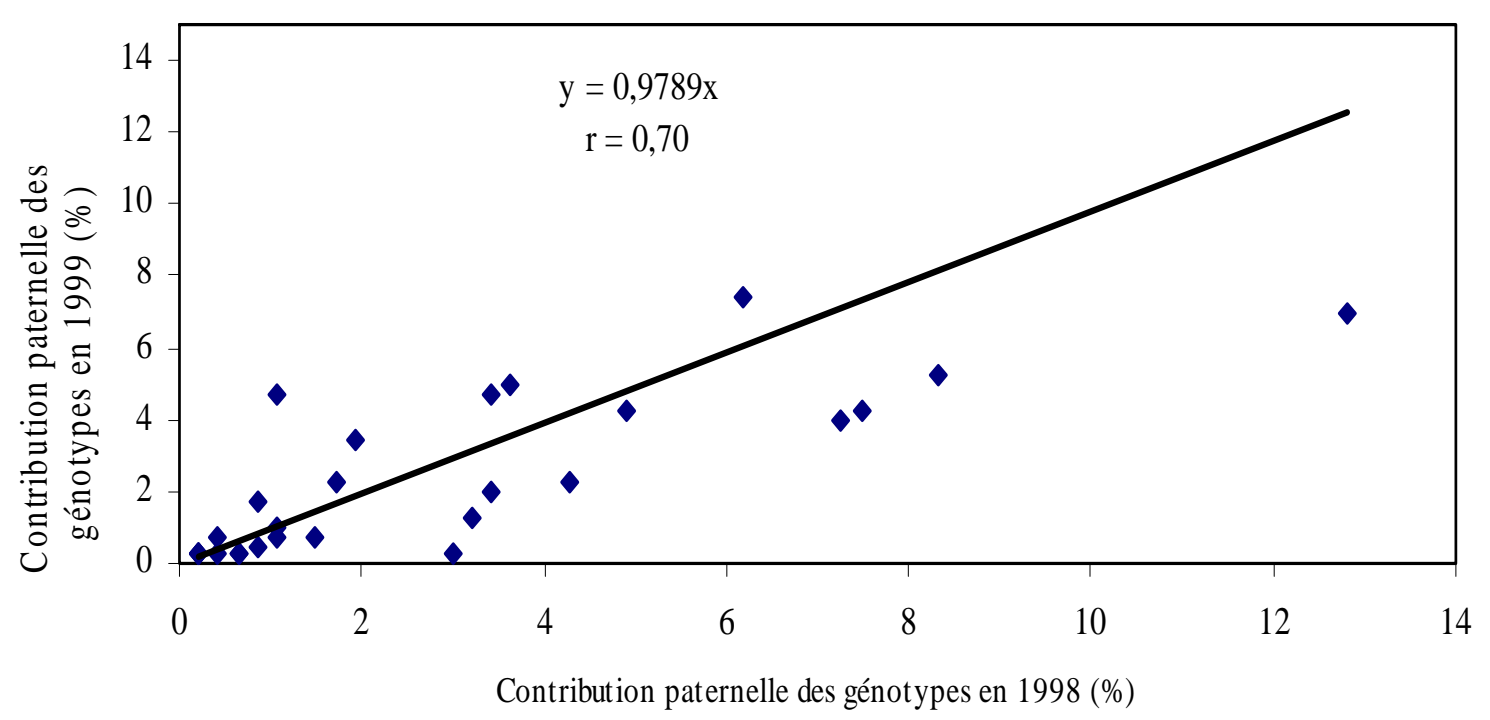

Figure 4 : Relation entre les contributions paternelles des génotypes d'hévéa sur 2 années

Linear correlation between the paternal contribution of the rubber tree genotyps over a 2-year period. 
Tableau 5 : Répartition de géniteurs mâles de Hevea brasiliensis dans différentes classes de pollinisateurs définies selon leur contribution paternelle

Males parents of Hevea brasiliensis distribution in pollination classes according to their paternal contribution

\begin{tabular}{|c|c|c|c|c|c|c|c|}
\hline \multirow[b]{2}{*}{ Classes } & \multirow[b]{2}{*}{$\begin{array}{l}\text { Contributions } \\
\text { mâles }\end{array}$} & \multicolumn{3}{|c|}{ Géniteurs mâles de 1998} & \multicolumn{3}{|c|}{ Géniteurs mâles de 1999} \\
\hline & & Total & AM1 & AM2 & Total & AM1 & AM2 \\
\hline $\begin{array}{l}\text { Faibles } \\
\text { pollinisateurs }\end{array}$ & ] $0 \% \quad 2 \%[$ & $\begin{array}{c}18 \\
(56,25 \%)\end{array}$ & $\begin{array}{c}11 \\
(92 \%)\end{array}$ & $\begin{array}{c}7 \\
(35 \%)\end{array}$ & $\begin{array}{c}26 \\
(65 \%)\end{array}$ & $\begin{array}{c}14 \\
(87,50 \%)\end{array}$ & $\begin{array}{c}12 \\
(50 \%)\end{array}$ \\
\hline $\begin{array}{l}\text { Pollinisateurs } \\
\text { Moyens }\end{array}$ & {$[2 \% 5 \%[$} & $\begin{array}{c}7 \\
(21,88 \%)\end{array}$ & $\begin{array}{c}0 \\
(0 \%)\end{array}$ & $\begin{array}{c}7 \\
(35 \%)\end{array}$ & $\begin{array}{c}9 \\
(22,5 \%)\end{array}$ & $\begin{array}{c}1 \\
(6,25 \%)\end{array}$ & $\begin{array}{c}8 \\
(33 \%)\end{array}$ \\
\hline $\begin{array}{l}\text { Grands } \\
\text { pollinisateurs }\end{array}$ & {$\left[\begin{array}{ll}5 \% & 10 \%[\end{array}\right.$} & $\begin{array}{c}5 \\
(15,63 \%)\end{array}$ & $\begin{array}{c}1 \\
(8 \%)\end{array}$ & $\begin{array}{c}4 \\
(20 \%)\end{array}$ & $\begin{array}{c}4 \\
(10 \%)\end{array}$ & $\begin{array}{c}1 \\
(6,25 \%)\end{array}$ & $\begin{array}{c}3 \\
(12,5 \%)\end{array}$ \\
\hline $\begin{array}{l}\text { Très grands } \\
\text { Pollinisateurs }\end{array}$ & {$[10 \%[$} & $\begin{array}{c}2 \\
(6,25 \%)\end{array}$ & $\begin{array}{c}0 \\
(0 \%)\end{array}$ & $\begin{array}{c}2 \\
(10 \%)\end{array}$ & $\begin{array}{c}1 \\
(2,5 \%)\end{array}$ & $\begin{array}{c}0 \\
(0 \%)\end{array}$ & $\begin{array}{c}1 \\
(1,5 \%)\end{array}$ \\
\hline Total & & $\begin{array}{c}32 \\
(100 \%)\end{array}$ & $\begin{array}{c}12 \\
(100 \%)\end{array}$ & $\begin{array}{c}20 \\
(100 \%)\end{array}$ & $\begin{array}{c}40 \\
(100 \%)\end{array}$ & $\begin{array}{c}16 \\
(100 \%)\end{array}$ & $\begin{array}{c}24 \\
(100 \%)\end{array}$ \\
\hline
\end{tabular}

Les valeurs entre parenthèses représentent le pourcentage des géniteurs dans la classe de pollinisateurs

The values in brackets represent the percentage of pollinisators class

Tableau 6: Test d'équilibre des contributions paternelles de génotypes pollinisateurs de Hevea brasiliensis en 1998.

Paternal contribution equilibrium test of pollinator genotypes of Hevea brasiliensis in 1998.

\begin{tabular}{|c|c|c|c|c|c|c|c|c|}
\hline Familles analysées & $\begin{array}{l}\text { Code du } \\
\text { génotype }\end{array}$ & Arbre-mères & $\begin{array}{l}\text { Nombre de } \\
\text { descendants }\end{array}$ & $\begin{array}{c}\text { Nombre de } \\
\text { parents mâles } \\
\text { observés } \\
\left(P_{o b}\right) \\
\end{array}$ & $\begin{array}{c}\text { Probabilité d’avoir } \\
2 \text { plein-frères } \\
\left(\sum \mathrm{f}^{2} j\right)\end{array}$ & $\begin{array}{c}\text { Nombre de } \\
\text { parents mâles } \\
\text { théoriques } \\
\left(P_{e f}\right) \\
\end{array}$ & $P_{e f} / P_{o b}$ & Khi2 \\
\hline \multirow{6}{*}{ Arbre-mère } & 115 & $3-14-6$ & 20 & 8 & 0,18 & 5,55 & 0,69 & 3,54 \\
\hline & 118 & $3-10-17$ & 24 & 9 & 0,15 & 6,70 & 0,74 & 0,78 \\
\hline & 221 & $3-11-14$ & 35 & 10 & 0,14 & 7,08 & 0,71 & 1,20 \\
\hline & 222 & $3-11-12$ & 28 & 12 & 0,11 & 9,11 & 0,76 & 0,9 \\
\hline & 300 & $3-4-6$ & 44 & 13 & 0,16 & 6,37 & 0,49 & 6,90 \\
\hline & & & & & & & Total & $13,3 \mathrm{~ns}$ \\
\hline \multirow{7}{*}{$\begin{array}{l}\text { Génotype (au moins } \\
2 \text { arbres-mères) }\end{array}$} & 115 & 3 & 38 & 9 & 0,19 & 5,31 & 0,59 & 2,58 \\
\hline & 118 & 2 & 37 & 12 & 0,18 & 5,56 & 0,46 & 7,45 \\
\hline & 140 & 5 & 58 & 17 & 0,14 & 7,15 & 0,42 & 13,56 \\
\hline & 221 & 2 & 58 & 14 & 0,10 & 9,56 & 0,68 & 2,06 \\
\hline & 300 & 3 & 79 & 17 & 0,14 & 7,15 & 0,42 & 13,56 \\
\hline & & & & & & & Total & $39,21 * *$ \\
\hline & & $\begin{array}{c}\text { Tous les plants } \\
\text { analysés en } \\
1998 \\
\end{array}$ & 467 & 32 & 0,06 & 15,74 & 0,49 & 16,79 \\
\hline
\end{tabular}

ns : Khi2 non significatif ; ** : Khi2 hautement significatif à $1 \%$

ns : Khi2 test no significant ; ** : Khi2 test higly significant at $1 \%$ 
Tableau 7 : Test d'équilibre des contributions paternelles de génotypes pollinisateurs de Hevea brasiliensis en 1999

Paternal contribution equilibrium test of pollinator genotypes of Hevea brasiliensis in 1999.

\begin{tabular}{|c|c|c|c|c|c|c|c|c|}
\hline $\begin{array}{l}\text { Familles } \\
\text { analysées }\end{array}$ & $\begin{array}{l}\text { Code du } \\
\text { génotype }\end{array}$ & Arbre-mères & $\begin{array}{l}\text { Nombre de } \\
\text { descendants }\end{array}$ & $\begin{array}{l}\text { Nombre de parents } \\
\text { mâles observés }\left(P_{o b}\right)\end{array}$ & $\begin{array}{c}\text { Probabilité } \\
\text { d'avoir } 2 \text { plein- } \\
\text { frères }\left(\sum \mathrm{f}^{2} j\right)\end{array}$ & $\begin{array}{c}\text { Nombre de } \\
\text { parents mâles } \\
\text { théoriques } \\
\left(P_{e f}\right)\end{array}$ & $P_{e f} / P_{o b}$ & Khi2 \\
\hline \multirow{8}{*}{$\begin{array}{l}\text { un arbre- } \\
\text { mère }\end{array}$} & 140 & $3-8-19$ & 30 & 12 & 0,12 & 8,33 & 0,69 & 1,62 \\
\hline & 140 & $3-16-9$ & 30 & 17 & 0,09 & 11,25 & 0,66 & 2,94 \\
\hline & 206 & $3-5-4$ & 56 & 11 & 0,23 & 4,42 & 0,4 & 9,79 \\
\hline & 206 & $3-7-20$ & 43 & 13 & 0,26 & 3,89 & 0,3 & 21,33 \\
\hline & 210 & $3-1-4$ & 66 & 14 & 0,17 & 5,81 & 0,41 & 11,54 \\
\hline & 221 & $3-15-1$ & 63 & 15 & 0,12 & 8,54 & 0,57 & 4,88 \\
\hline & 300 & 3-4-6 & 51 & 12 & 0,27 & 3,7 & 0,32 & 18,62 \\
\hline & & & & & & & Total & $70,72 * *$ \\
\hline \multirow{5}{*}{$\begin{array}{l}\text { Génotype } \\
\text { (plus d'1 } \\
\text { arbre-mère) }\end{array}$} & 140 & 2 & 60 & 20 & 0,09 & 11,32 & 0,566 & 6,66 \\
\hline & 206 & 5 & 157 & 21 & 0,19 & 5,14 & 0,245 & 48,94 \\
\hline & 300 & 3 & 79 & 17 & 0,14 & 7,15 & 0,290 & 10,60 \\
\hline & & & & & & & Total & $66,20 * *$ \\
\hline & & $\begin{array}{c}\text { Tous les plants } \\
\text { analysés en } \\
1999 \\
\end{array}$ & 404 & 40 & 0,08 & 11,92 & 0,30 & 66,15 \\
\hline
\end{tabular}

** Khi2 hautement significatif à $1 \%$

** Khi2 test higly significant at $1 \%$

\section{DISCUSSION}

La contribution paternelle ou «succès reproducteur mâle» a été l'objet de plusieurs études en pollinisation naturelle puisqu'elle constitue un caractère important dans l'amélioration des plantes (Fast et al., 1986 ; Schoen et Steward, 1987). Dans le verger à graines étudié, plusieurs génotypes ont participé à la pollinisation des arbres échantillonnés. En 1998 et en 1999, 32 et 40 géniteurs mâles sur 51 ont respectivement participé à la création de 467 et 404 descendants. L'implication d'un nombre aussi élevé de géniteurs mâles dans la pollinisation des arbres du verger à graines, constitue à priori, un avantage du fait de la diversité génétique que cela pourrait entraîner dans les descendances. Cependant, cette étude a mis en évidence un déséquilibre de contribution paternelle des génotypes d'hévéa. La pollinisation a été particulièrement dominée par 4 clones dont le cumul des contributions paternelles, dans l'ensemble de plantules analysées, a atteint $40 \%$ quelle que soit l'année. Des résultats similaires ont été observés par Razafimaharo (2000) entre trois introductions en étudiant le flux de gènes dans un verger à graines d'eucalyptus (Eucalyptus grandis) à Madagascar. Cet auteur (Razafimaharo, 2000) a suggéré que l'étalement de la floraison des arbres (c'est-à-dire la phénologie), pourrait expliquer ses résultats. L'étude de la paternité de Streiff (1998) portant sur une population naturelle de chêne vert a permis de conclure que les géniteurs mâles ont contribué inégalement à la formation des descendances. Comme Razafimaharo (2000), cet auteur pense que le synchronisme de floraison des arbres et la quantité de fleurs produites seraient responsables de ces inégalités.

En plus de la phénologie, Streiff (1998) a justifié ses résultats par l'influence de la distance qui sépare les arbres-mères des pollinisateurs sur la contribution paternelle et le système d'incompatibilité refoulant préférentiellement le pollen de certains arbres chez le chêne. Yacine et Lumaret (1988) ont trouvé l'explication des inégalités des contributions paternelles chez le chêne vert dans la différence de la fonction reproductive mâle des arbres. Ces auteurs ont suggéré que les arbres ne sont pas nécessairement pollinisés par leurs voisins, mais par ceux qui leur sont phénologiquement synchrones et qui ont prioritairement développés des aptitudes à la fécondation. Dans notre étude, 
la contribution paternelle d'un même génotype d'hévéa est restée stable quelle soit l'année ; elle semble donc être une caractéristique génotypique comme l'ont suggèré Yacine et Lumaret chez le chêne vert (1988). Pourtant en pollinisation manuelle de l'hévéa, aucun effet lié au géniteur mâle n'a été mis en évidence (Legnaté et Clément-Démange, 1991a). La méthode utilisée en pollinisation artificielle, qui consiste à mettre le pollen d'un seul génotype sur les ovules de son partenaire femelle n'a pas induit de compétition entre les géniteurs mâles. Dans ces conditions, le taux de fécondation estimé en moyenne à $3 \%$ est variable selon le géniteur femelle (Hamzah et al., 2002). Ce qui serait loin d'être le cas en pollinisation naturelle où les ovules reçoivent un mélange de pollen de différents pollinisateurs (Thomson et Thomson 1989 ; Dudast et Ritland 1991). Par conséquent, comme il n'existerait aucune barrière reproductive entre différents clones d'hévéa (Vrancken, 1981), seuls les génotypes dont le pollen germe plus rapidement que les autres va féconder en priorité les fleurs femelles. Le pollen des génotypes à forte contribution paternelle dans le verger à graines, serait donc doté d'une plus grande faculté germinative que celui des autres génotypes. Chez l'hévéa, des différences interclonales significatives au niveau de la germination du pollen, en relation avec leur teneur en eau ont été mises en évidence in vitro (Dijkman 1938; Vrancken 1981). Sur le même milieu de culture, le pollen d'hévéa le plus déshydraté germe plus rapidement que le pollen le moins déshydraté (Vrancken, 1981). La déshydratation du pollen assure donc sa maturité et lui confère un pouvoir fécondant. Ainsi, les meilleurs pollinisateurs du verger à graines se caractériseraient par un pollen plus déshydraté que celui des génotypes à faible contribution paternelle. Des essais de germination de pollen in vitro et en conditions naturelles serait cependant nécessaire pour confirmer ces hypothèses. Toutefois, la rapidité de germination du pollen semble être à la base de contributions paternelles inégales chez l'épicéa commun ou Picea abies (Aronen et al., 2002).

Dumas et al. (1984) ont montré que l'acceptation du pollen par le stigmate des angiospermes se fait grâce à un mécanisme de reconnaissance pollen-pistil. L'acceptation du pollen par le pistil serait une réaction de type «immunitaire» entre des molécules-signaux du complexe génétique $S$, qui sont en fait des lectines portées par le pollen et des molécules-réceptrices sur le stigmate. Ceci serait la preuve que les meilleurs pollinisateurs portent un nombre important de molécules-signaux facilement reconnaissables, qui saturent rapidement les sites récepteurs des stigmates des fleurs des arbres qu'ils pollinisent. En effet, cette étude, a montré, à travers une corrélation positive et significative, que les géniteurs mâles, à bonne contribution paternelle, ont participé à la pollinisation de plusieurs arbres par rapport aux autres.

Les déséquilibres de contribution paternelle, notés au niveau des descendances, ont induit des proportions élevées de pleins-frères de géniteurs mâles à forte contribution paternelle. Ces déséquilibres de contribution paternelle seraient la cause de croisements non panmictiques, dont l'incidence sur la sélection en verger à graines peut être importante. En effet, pour évaluer les géniteurs en pollinisation libre dans un verger à graines sur le test de descendants, comme cela se fait en sélection recurrente, la proportion de pleins-frères doit être faible, de sorte à considérer les familles comme des familles équilibrées de demi-frères (Baradat, 1982). Mais, les résultats ont montré que le régime de reproduction de l'hévéa en verger à graines n'a pas été panmictique puisque les contributions paternelles ont été variables entre les génotypes étudiés. Le groupe de génotypes, à bonne contribution paternelle révélé par cette étude, a déséquilibré la constitution des familles. Ainsi, les évaluations en croisement des génotypes de l'essai refléteront plus la valeur en croisement entre les arbres de l'essai et ce groupe de génotypes, à bonne contribution paternelle. Cette situation, fait que les conclusions sur l'évaluation des géniteurs du verger à graines sont erronées. Par conséquent, la sélection en verger à graines de génotypes d'hévéa pourrait se révéler très peu efficace pour l'amélioration globale des populations sauvages.

\section{CONCLUSION}

L'étude de la contribution paternelle des génotypes sauvages d'hévéa en pollinisation libre en verger à graines, a permis d'évaluer le brassage génétique entre les arbres utilisés. Si la proportion de génotypes ayant participé à la pollinisation est satisfaisante (42 génotypes sur 50) cette étude a malheureusement montré une grande variabilité de la contribution paternelle entre les génotypes pollinisateurs. La plupart 
des génotypes ont présenté une faible contribution paternelle sur les 2 années d'étude. Parmi les pollinisateurs recensés, les génotypes du groupe AM2 ont été plus nombreux et plus efficaces que ceux du groupe AM1 grâce à 4 génotypes qui ont particulièrement dominé la pollinisation. Cette différence de pollinisation notée entre les génotypes indique un mode de reproduction non panmictique qui diminuerait l'efficacité de la sélection en verger à graines dans la population sauvage. Ainsi, le groupe de meilleurs pollinisateurs pourrait être utilisé comme donneur de pollen dans une évaluation de type top-cross, où seuls les arbres pollinisés seraient testés. Une amélioration substantielle des progrès génétiques pourrait être obtenue si les microsatellites étaient utilisés dans l'analyse de la paternité en vue détablir des plans de croisements. Ainsi, les 2 parents seraient connus pour chaque descendant issu de pollinisation libre comme en pollinisation dirigée. $\mathrm{Ce}$ qui faciliterait l'estimation des valeurs génétiques et augmenterait ainsi l'efficacité de la sélection en verger à graines.

\section{REFERENCES}

Aronen T., Nikkanen T., Harju A., Tiimonen H. et $\mathrm{H}$. Häggman. 2002. Pollen competition and seed-siring success in Pecea abies. Theor Appl Genet . 104 : 638 - 642.

Baradat P. 1982. Génétique quantitative. Modèles statistiques et génétiques de base. fascicule I. INRA Bordeaux (France), $203 \mathrm{p}$.

Baradat P., Marpeau A. et B. Dagan. 1984. Les terpènes du pin maritime ; aspects génétiques et biologiques. Estimation du taux moyen d'autofécondation et mise en évidence d'écart à la panmixie dans un verger à graines de semis. Ann.Sci.For. 41 (2) : $107-134$.

Baciellerie R. 1994. Régime de reproduction et flux de gènes dans un peuplement mixte de chêne sessile (Quercus petraea) et de chêne pédonculé (Quercus robur). Thèse de doctorat, Université de Montpellier II (France), $88 \mathrm{p}$.

Baudoin L., Baril C., Clément-Demange A., Leroy T. et D. Paulin. 1997. Recurrent selection of tropical tree crops. Euphytica $96: 101-114$.
Chevallier M.H. 1988. Genetic variability of Hevea brasiliensis germplasm using isozyme markers. J. nat.Rub.Res. 3 (1) : 42 - 53.

Clement-Demange A., Nicolas D., Legnaté H., Rivano F., Le Guen V., Gnagne M. et T. Chapuset. 1995. Hevea, stratégies de sélection. Recherche-Développement. 2 (3) : 5 - 18.

Clement-Demange A., Legnate $H$., Seguin M., Boutry M., Leconte A., Luo H., Chapuset T., Pinard F., Doumbia A., Gobina S. et K. E. Koffi. 1997. Etude et caractérisation de nouvelles ressources génétiques : Leur utilisation en amélioration de l'hévéa. Rapport scientifique final. volume $3 / 3$. CIRAD et CNRA Bimbresso, 96 p.

Djikman M. J. 1938. Voorloopie gegevens over het bewaren von hevea.Stuifmel. Arch. Rubbercult. 22 : 239 - 259.

Dudast M. R. et K. Ritland. 1991. Multiple paternity and sel-fertilization in relation to flora age in Mimulus guttatus Am. J. Bot. 78 : 1746 1753.

Dumas C., Andrienne E., Clark R. et B. Knox. 1984. $\mathrm{La}$ fécondation des fleurs. La Recherche, 15 (161) : 1518 - 1526.

Fast W., Dancik B. P. and R. C. Bower. 1986. Mating system and pollen contamination in a Douglas clone bank. Canadian journal of forest research $16: 1314-1319$.

Hamzah S., Chan J. L. and H. Y. Yeang. 2002. Pollen tube growth and fruit-set success in Hevea brasiliensis hand-pollination influenced by the choice of clone and female flower. Euphytica $123: 1$ - 8.

Ho C. Y. 1979. Conservation and utilization of Hevea germplasm. In : Malays.Appl.Biol. 8 (1) : 1 - 14.

Leconte A. 1984. La reproduction sexuée de l'Hevea brasiliensis : Une approche histologique et expérimentale. Doctorat $3^{\mathrm{e}}$ cycle. Université de Montpellier II Sciences et techniques du Languedoc (France), $68 \mathrm{p}$.

Legnate $H$. et A. Clement-Demange. 1991a. Pollinisation artificielle et Amélioration génétique de l'hévéa en Côte d'Ivoire. Caoutchouc et Plastiques 136 (91) : 99 - 103.

Legnate $H$. et A. Clement-Demange. 1991 b. Evaluation et choix des géniteurs de Hevea brasiliensis en Côte d'Ivoire. Caoutchouc et plastiques 137 (91) : 114 - 118 .

Marshall T. C., Slate J., Kruuk B. et E. Pemberton. 1998. Statistical confidence for likelihood- 
based paternity inference in natural populations. Molecular Ecology, 7 : $639-655$

Meagher T. R. 1986. Analyse of paternity within a natural population of Chamaelirum luteum. I. Identification of most-likely male parents. The American Naturalist, 128 : 199 - 215.

Schoen D. J., and S. C. Stwart. 1987. Variation in male reproductive investment and male reproductive success in white spruce. Evolution 40 : 1109 - 1120.

Streiff R. 1998. Structure génétique spatiale et flux polliniques dans une chenaie mixte : Quercus petraea (Matt.) LIEBL., Quercus Robur L. Thèse de Doctorat, Université de Montpellier II Sciences et Techniques du Languédoc (France), 84 p.

Razafimaharo V. 2000. Analyse des flux géniques par pollen à l'aide de marqueurs microsatellites dans un verger à graines d'Eucalyptus grandis à Madagascar. Mém. DEA. Université d'Antananarivo. Département forêt, $40 \mathrm{p}$.
Thompson E. 1975. The estimation of perwise relationships. Annals of human genetics, $39: 173-188$

Thompson E. 1976. Inference of genealogical structure. Social science information, 15 : $477-526$

Thomson J. D. and B. A. Thomson 1989. Dispersal of Erythronium grandiflorum pollen by bumblebees : implication for gene flow and reproductive success. Evolution, 43 : $657-661$.

Vrancken E.. J. M. 1981. Quelques aspects sur la biologie florale et la conservation du pollen d'Hevea brasiliensis. Rapport de recherche. Université de Montpellier II. Sciences Techniques du Languédoc (France)/ IRCA, $38 \mathrm{p}$.

Yacine A. et R. Lumaret 1988. Distribution spatiale des génotypes dans une population de chêne vert (Quercus ilex L.), flux génique et régime de reproduction. Génet. Sél. Evol., $20(2)$ : 181 - 198. 